\title{
A Minimal-Invasive Exosome Spray Repairs Heart After Myocardial Infarction
}

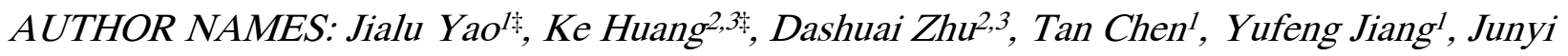

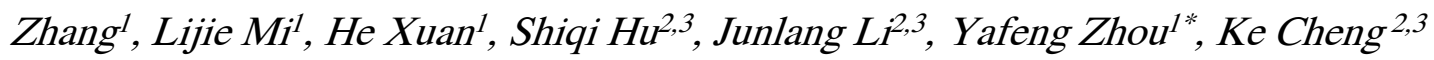

\section{AUTHOR ADDRESS:}

${ }^{1}$ Department of Cardiology, Dushu Lake Hospital Affiliated to Soochow University, Medical Center of Soochow University, Suzhou Dushu Lake Hospital, Suzhou 215000, China.

${ }^{2}$ Department of Molecular Biomedical Sciences and Comparative Medicine Institute, North Carolina State University, Raleigh, North Carolina 27607, USA.

${ }^{3}$ Joint Department of Biomedical Engineering, University of North Carolina at Chapel Hill \& North Carolina State University, Raleigh, North Carolina 27607, USA.

\section{Corresponding Authors:}

zhouyafeng73@126.com

†These authors contributed equally.

Competing interests: The authors declare that they have no competing interests. Data and materials availability: All data needed to evaluate the conclusions in the paper are present in the paper and/or the Supplementary Materials. Additional data related to this paper may be requested from the authors. 


\section{SUPPLEMENTARY MATERIALS AND METHODS}

\section{Cardiomyocyte proliferation with EXOS}

To determine the proliferative potential of EXOS to cardiomyocytes, we cultured NRCMs as aforementioned as the control. To distinguish if each component of EXOS has impact on cardiomyocytes, NRCMs with scaffold only or with same dose of exosomes (without fibrin vehicle) were also added as controls. After 48 hours of coculture, NRCMs were fixed with 4\% PFA (paraformaldehyde solution), permeabilized and blocked with Dako solution that contains 0.01\% saponin (Sigma-Aldrich), and then incubated with primary antibodies overnight at $4^{\circ} \mathrm{C}$. TUNEL Assay (G3250, Promega) was done before primary antibody incubation: anti-Ki67 (1:200; ab15580, abcam), anti-aroraB (1:200; ab2254, abcam), anti-sarcomeric alpha actinin (1:200; ab9465, abcam) and anti-pH3 (1:200, ab24684, abcam). Goat anti-Mouse IgG (Alexa Fluor 488, ab150113, abcam) or Goat anti-rabbit IgG (Alexa Fluor 594, ab150080, abcam) were used as secondary antibodies with primary antibodies for 1.5 hour at room temperature. Samples were then mounted in Prolong Gold with DAPI (P36935, Thermo Fisher). Images were taken by confocal fluorescent microscope (ZEISS LSM 880), and then characterized from ZEN lite and the NIH ImageJ software.

Mouse model of MI

5-8-week-old C57BL/6 mouse (Charles River Laboratories) were anesthetized with a 1.5\% isofluorane-oxygen mixture under artificial ventilation with a rodent ventilator (SAR-1000 Small Animal Ventilator). MI was induced under sterile surgical conditions as previously described. ${ }^{23}$ Briefly, an acute MI was developed by LAD permanent ligation (6-0 prolene blue 30", Esutures) 
through a left thoracotomy at the 4th intercostal space. The heart was randomized to receive one of the following 4 treatments: i) MI induction only; ii) MI induction with spray of scaffold onto the cardiac infarcted area; iii) MI induction with exosome myocardial injection; or iv) MI induction with EXOS spray on the surface of the discolored infarcted myocardium. After completion of surgery, mice were extubated and observed for about 10 minutes until they were able to move. All animals were euthanized 28 days after MI.

Tissue Immunohistochemistry (IHC)

Tissue IHC staining was performed as previously described. ${ }^{23}$ Briefly, heart cryosections were fixed with 4\% paraformaldehyde solution, blocked and permeabilized with Dako solution that contains $0.01 \%$ saponin (Sigma-Aldrich), and then incubated with primary antibodies overnight at $4{ }^{\circ} \mathrm{C}$ : rabbit anti- von Willebrand factor (1:200, ab6994, Abcam); mouse anti-alpha sarcomeric actin (1:200, a7811, Sigma); rabbit anti-Ki67 (1:200, ab15580, Abcam); rabbit antipH3 (1:200, ab5176, Abcam); and rabbit anti- AuroraB (1:200, ab2254, Abcam). Goat anti-Mouse IgG (Alexa Fluor 488, ab150113, abcam) or Goat anti-rabbit IgG (Alexa Fluor 594, ab150080, abcam) were used as secondary antibodies for a 1.5-hour incubation with primary antibodies at room temperature. Then, samples were mounted in Prolong Gold antifade mountant with DAPI (ThermoFisher). All images were taken with confocal fluorescent microscope (ZEISS LSM 880). Tissue morphology was characterized by images using ZEN lite and the NIH ImageJ software.

Cardiac functional assessment

To measure mice cardiac dimensions and function, echocardiography was performed at $4 \mathrm{~h}$ and 28 days after MI surgical operation following induction of anesthesia with a $1.5 \%$ isofluorane- 
oxygen mixture. Echocardiography was performed with a Philips Cx-30 Ultrasound System with a Philips L15-7io high frequency probe. Two-dimensional long axis images were record from the left caudal (apical) view. Two-dimensional guided M-mode images at chordae tendineae level were evaluated. M-mode measurements of left ventricle end-diastolic and end-systolic dimensions (LVEdD and LVEsD, respectively) were performed by using the leading-edge method of the American Society of Echocardiography. ${ }^{59}$ For estimation of each parameter, the average of three measurements from three different cycles in an image was obtained. Left ventricular end-diastolic and systolic volumes (LVEdV and LVEsV, respectively) were calculated by the biplane method of disks (modified Simpson's rule). Left ventricular ejection fraction (LVEF) was determined by using $($ LVEdV-LVEsV/LVEdV) $\times 100 \%$, and fractional shortening $(\mathrm{FS})$ was calculated from the M-mode echocardiography images as (LVEdD-LVEsD/LVEdD) $\times 100 \%$.

Heart morphologic studies

After all animals were euthanized, hearts from mice were collected and frozen in optimum cutting temperature (OCT) compound (Tissue-Tek, Torrance, CA) after equilibration with increasing sucrose solutions up to $30 \%$ overnight. Hearts were then snap-frozen in liquid nitrogen, and cryo-sectioned with a thickness of $5 \mu \mathrm{m}$ from the apex to the ligation level with $100 \mu \mathrm{m}$ intervals for Masson's Trichrome staining. Masson's trichrome staining was performed as described in previous studies. ${ }^{60,61}$ Images were acquired with a PathScan Enabler IV slide scanner (Advanced Imaging Concepts). From the Masson's trichrome images, morphometric parameters including viable myocardium and infarct thickness were measured in each section with ImageJ software. 
Thoracoscope guided minimal-invasive spray of EXOS on a rat heart

5-7-week-old SD rats (Charles River Laboratories) were anesthetized with a 2.5\% isofluorane-oxygen mixture under artificial ventilation with a rodent ventilator (SAR-1000 Small Animal Ventilator). MI was induced under sterile surgical conditions as previously described. ${ }^{23} 6$ animals randomly received either exosome myocardial injection or minimal-invasive EXOS spray ( $n=3$ in each group). Two small incisions (diameter $<0.5 \mathrm{~cm}$ ) were opened near mid clavicular line at the fourth intercostal space. Mini endoscope was inserted into one of the incisions for guidance of a precision tweezer to tear the pericardium. Then, the EXOS spray needle was guided to spray after the tweezer was pulled out. After completion of thoracoscopy, rats were extubated and observed for about 15 minutes until they were able to move. We pre-labeled exosomes with DiD (1,1'-dioctadecyl-3,3,3', 3'-tetramethylindodicarbocyanine, 4-chlorobenzenesulfonate salt, D7757, ThermoFisher) and then prepared EXOS. All rats were euthanized, and the hearts were harvested 24 hours after treatment. EXOS retention were analyzed through Xenogen IVIS imaging system (Caliper Life Sciences, Mountain View, CA).

\section{A DAPI DiD}
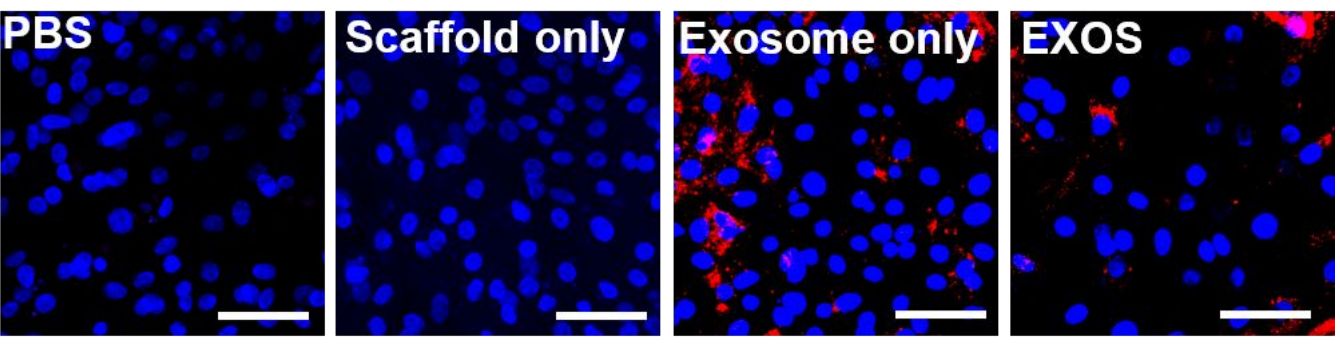

B DAPI TUNEL
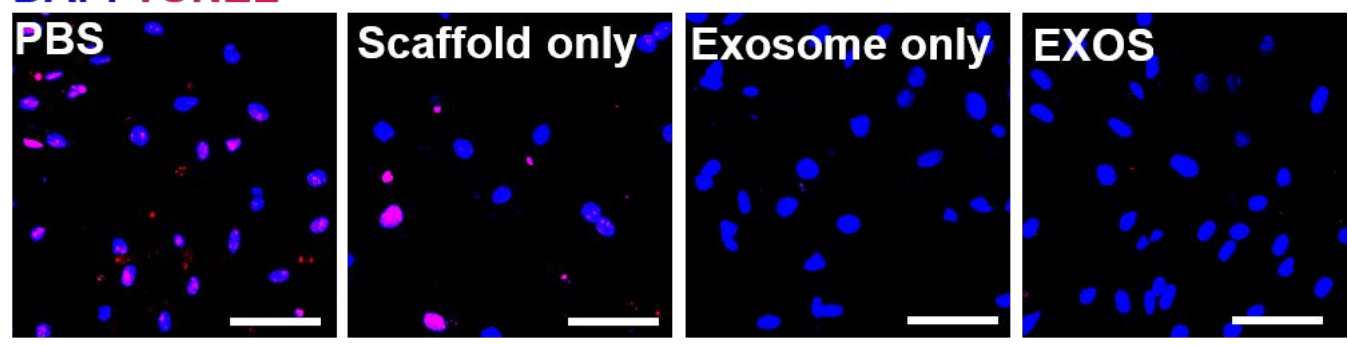
Supplementary Figure 1. Exosome uptake and cell apoptosis in cardiomyocytes after EXOS application in vitro. (A) Representative 2-color fluorescence images showing the uptake of DiD prelabeled exosomes (red) in cardiomyocytes, including PBS control, scaffold only control, exosome only control, and EXOS groups. Scale bars, $50 \mu \mathrm{m}$. (B) TUNEL essay fluorescence images showing the apoptosis (red) of cardiomyocytes in different groups, including PBS control, Empty EXOS control, exosome only control, and EXOS groups. Scale bars, $50 \mu \mathrm{m}$.

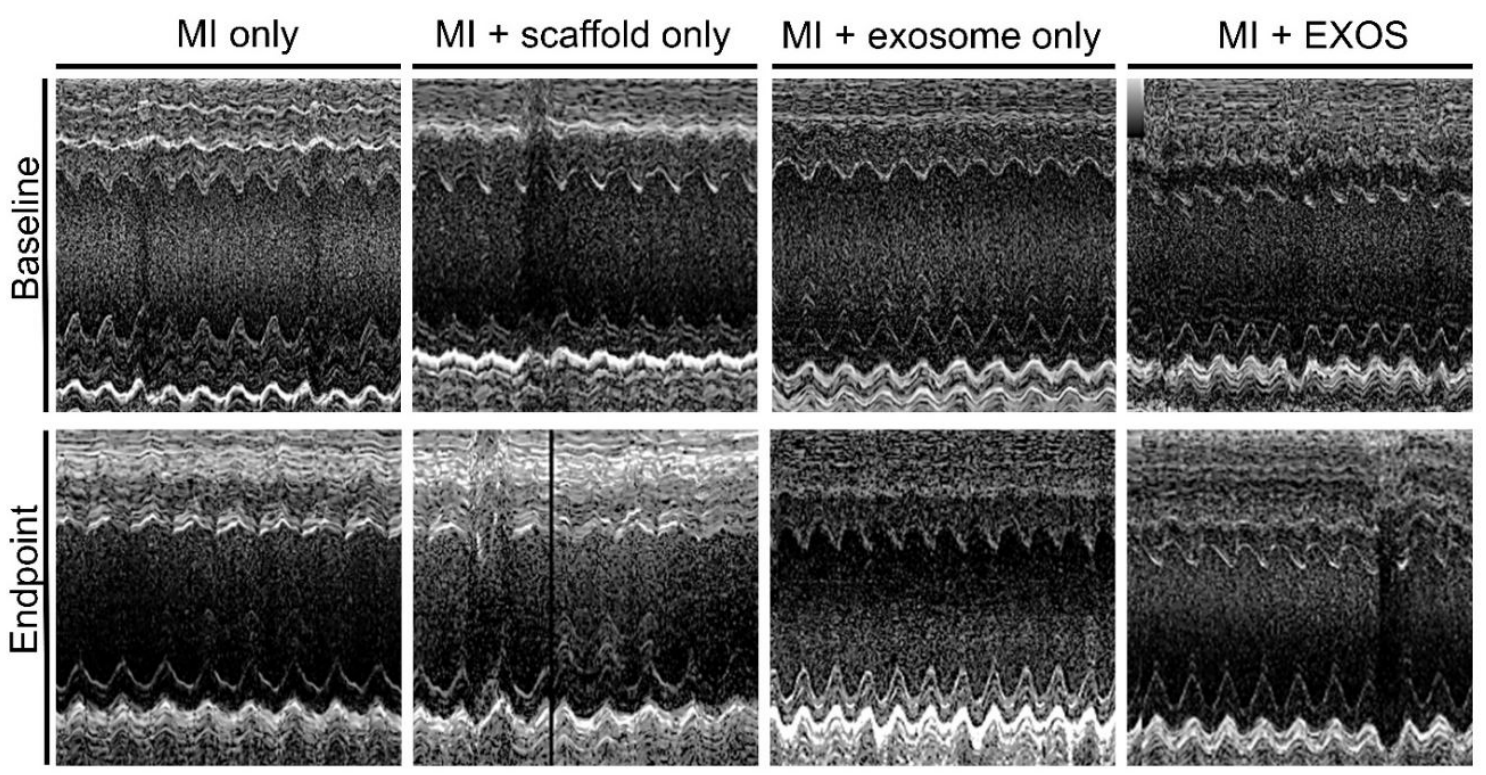

Supplementary Figure 2. Representative echocardiograms in each group at both baseline and endpoint.

Supplementary Table 1. Group allocation for the animal studies. 


\begin{tabular}{ccccc}
\hline $\begin{array}{c}\text { Group } \\
\text { No. }\end{array}$ & Group name & Surgery & Treatment & $\begin{array}{c}\text { N } \\
\text { number }\end{array}$ \\
\hline 1 & Healthy control & N/A & N/A & 6 \\
\hline 2 & Ml injury control & MI & PBS & 6 \\
\hline 3 & Ml injury with exosome injection & MI & Exosome myocardial injection & 6 \\
\hline 4 & Ml injury with scaffold spray & MI & Scaffold only & 6 \\
\hline 5 & Ml injury with EXOS & MI & EXOS & 6 \\
\hline
\end{tabular}

Supplementary Video. Thoracoscope guided minimal-invasive EXOS spray on a rat heart. 